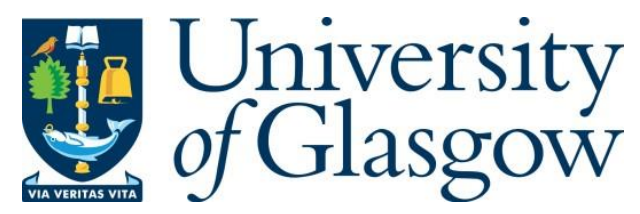

Nikolaou, C.K., and Lean, M.E.J. (2017) Mobile applications for obesity and weight management: current market characteristics. International Journal of Obesity, 41(1), pp. 200-202.

There may be differences between this version and the published version. You are advised to consult the publisher's version if you wish to cite from it.

http://eprints.gla.ac.uk/131031/

Deposited on: 20 February 2017

Enlighten - Research publications by members of the University of Glasgow http://eprints.gla.ac.uk 


\section{Mobile applications ('apps') for obesity and weight management: current market characteristics}

7

\section{Charoula K Nikolaou ${ }^{1}$, Michael E J Lean ${ }^{2}$}

Affiliations: ${ }^{1}$ Biogov, Universite Catholique de Louvain, Collège Thomas More, Louvain Belgium

${ }^{2}$ Human Nutrition, School of Medicine, University of Glasgow, Scotland

\section{Corresponding Author}

Charoula-Konstantia Nikolaou

Universite Catholique de Louvain

Collège Thomas More

Place Montesquieu 2

Louvain-La-Neuve

1348

E-mail: charoulanikolaou@yahoo.co.uk

Short Report Word count: Abstract $=200$ Main text $=1,444$

Key Words: apps, mHealth, weight-management, weight-gain prevention

Conflict of interest: The authors declare there is no conflict of interest.

Funding: No funding 
Abstract (word limit= 199: word count=200)

32

mHealth is the fastest-developing eHealth sector, with over 100,000 health-apps currently available. Overweight/obesity is a problem of wide public concern which is potentially treatable/preventable through mHealth. This study describes the current weightmanagement app-market.

37

Five app-stores (Apple, Google, Amazon, Windows, Blackberry) in UK, US, Russia, Japan, Germany, Italy, France, China, Australia, and Canada were searched for key words: 'weight', 'calorie', 'weight-loss', 'slimming', 'diet', 'dietitian' and 'overweight' in January/February 2016 using App-Annie (San Francisco, CA, USA) software. The ten most downloaded apps in the lifetime of an app were recorded. Developers' lists and the app descriptions were searched to identify any professional input with key words 'professional', 'dietitian', and 'nutritionist'.

A total of 28,905 relevant apps were identified; Apple-iTunes=8,559(4,634, 54\% paid), 46 Google-Play=1,762 (597, 33.9\% paid), Amazonapp=13,569 (4,821, 35.5\% paid), Windows= 2,419 (819, 17\% paid), Blackberry=2,596 (940,36\% paid). The 28,905 identified apps focused mainly on physical actity (34\%), diet (31\%), and recording/monitoring of exercise, calorieintake and body-weight (23\%). Only 17 apps(0.05\%) were developed with identifiable professional input.

51

52 Apps on weight-management are widely available and very popular but currently lack professional content-expertise. Encouraging app-development based on evidence-based

54 online approaches would assure content quality, allowing health-care professionals to 55 recommend their use. 


\section{Background (Short Report word limit=1,547: word count=1,500)}

58

59

60

\section{8}

Mobile-Health (mHealth), a subcategory of eHealth, covering interventions and practice involving 'apps' and mobile devices is a new and fast-growing field. Blackberry introduced the first mobile device with computing features in 2002, followed by Apple in 2007 and Google in $2008^{1}$. In 2010, 'tablet' computers whose portability, and large screens expanded the mobile market further, were introduced by Apple and Google ${ }^{1,2}$. Subsequent smartphones and tablets with advanced features and capabilities, coupled with falling prices, increased the ownership of these devices rapidly ${ }^{3}$. Unique subscriber-penetration currently stands at $79 \%$ and $45 \%$ of the entire population in the developed and developing worlds, respectively 4 .

'Apps' are software programs developed to run on mobile devices to accomplish a specific purpose $^{5}$. There are more than 100,000 $\mathrm{mHealth}$-apps available ${ }^{6}$ for downloading free or at a nominal value from five stores; Apple-iTunes for iOS operating systems, Google-Play for Android operating system, Amazonapp for Fire and Android operating systems, Windows and Blackberry for their eponymous operating systems ${ }^{7}$.

Between 1980-2013 the global prevalence of overweight (BMI>25 $\mathrm{kg} / \mathrm{m}^{2}$ ) increased from $29 \%$ to $37 \%$ in men and from $30 \%$ to $38 \%$ in women ${ }^{8}$. Obesity prevalences $\left(B M I>30 \mathrm{~kg} / \mathrm{m}^{2}\right.$ ) now approach or exceed $30 \%$ in US, UK, the Gulf States, Australia, and Canada ${ }^{8}$. In UK, obesity prevalence now reaches about $40 \%$ by age $65^{9}$ and national physical activity recommendations are met by $69.5 \%$ of adults ${ }^{10}$. The need for effective anti-obesity interventions that will reach large population-sectors at low-cost is increasingly critical. A recent systematic review concluded that self-directed interventions can promote weightloss $^{11}$. Mobile-apps on weight-management could provide low-cost, self-directed mechanisms to reach the $80 \%$ of young, obesity-prone, populations who own tablets or smartphones ${ }^{12}$. Healthcare professionals and public health services could suggest and reinforce the movement towards prescription of more evidence-based apps. This study quantifies and describes the current weight-management provision in the largest appmarkets worldwide. 
An electronic search was conducted to identify apps relevant to weight-management in the five main app-stores, covering all devices and operating systems currently on the market: Apple-iTunes, Google-Play, Amazonapp, Windows, and Blackberry. The app-stores in the leading ten app-markets worldwide (UK, US, Russia, Japan, Germany, Italy, France, China, Australia, and Canada) were searched using key-words: 'weight', 'calorie', 'weight-loss', 'slimming', 'diet', 'dietitian' and 'overweight'. App-Annie (San Francisco, CA, USA) software was used for Apple-iTunes and Google-Play stores, and the provided search engines for Windows, Amazonapp, and Blackberry stores. The software automatically converts English into the appropriate language for searching.

100

101

Developers' lists and descriptions of all apps identified as relevant to weight-management were searched with key-words: 'professional', 'dietitian' and 'nutritionist' in order to identify professional input for development, and evidence of development for professional bodies, universities or governmental Health Agencies. ANOVA on SPSS-23 was used to examine differences between countries and online shops.

106

The five free, and five paid, most downloaded apps from 'Lifestyle/Health' and 'Fitness' categories of the five app-stores for the same countries (total 500 apps) were identified for more complete description, using App-Annie (San Francisco, CA, USA) software.

\section{Results}

112

113 A total of 28,905 unique apps relevant to weight-management were identified; Apple114 iTunes=8,559 (4,634, 54\% paid), Google-Play=1,762 (597, 33.9\% paid), Amazonapp=13,569 115 (4,821, 35.5\% paid), Windows=2,419 (819, 17\% paid), Blackberry=2,596 (940, 36\% paid) 116 (Table 1). They accounted for over two billion downloads over the apps' lifetimes. However, 117 over half of all those downloads were generated by just 15 apps, 12 on physical activity and 118 three on monitoring of calorie intake, physical activity, body weight, and sleeping patterns. 119 Of these eleven apps were directly associated with a wearable device. 
121 The 28,905 identified apps focused mainly on physical activity (34\%), on diet (31\%), and on

122 recording and monitoring of exercise, calorie intake and body-weight (23\%) (Figure 1).

123 'Weight loss' or 'slimming' was specifically mentioned by 22,587 (78\%) of apps relevant to 124 weight-management. There were 53 apps aimed at prevention of diabetes, cancer or chronic125 disease that included weight maintenance in their content. However, none of the 28,905 126 apps mentioned specifically the words 'obesity-prevention' or 'prevent weight-gain'.

128 There were few differences between countries in the total numbers of apps available. In all 129 countries, Amazonapp store contained significantly more weight-management apps $130(p<0.001)$ and Apple iTunes had a largest proportion of paid apps $(p<0.001)$.

132 The most downloaded free and paid apps in 'Lifestyle/Health' and 'Fitness' categories listed 133 by the five app-stores in ten countries are shown in supplementary Table 1. In every country, 134 and in every store, at least one app directed towards weight-management was among the 135 top ten apps. In US, UK, Canada, Germany, and Russia all top ten apps were for weight136 management. The actual numbers of downloads of these apps was not provided by all app 137 stores.

139 Searching the developers' list and descriptions of the retrieved apps, only $17(0.05 \%$ of $14028,905)$ indicated that they were developed with the input from a professional, or for 141 professional bodies, universities or governmental health agencies. Five of these were designed for use by health-care professionals, rather than directly by the public.

144 Among the top ten apps in 'Lifestyle/Health' and 'Fitness' categories in the ten countries (500 apps in total), only three apps $(0.6 \%)$ were identified as having been developed by professional bodies.

\section{Discussion}

149 Interest and activity in mHealth is high. The total number of available mHealth apps is 150 growing rapidly, including an increase of $284 \%$ in the number of available mHealth apps since $1512013^{13}$. 
153 Over a quarter of approximately 100,000 mHealth apps are directed to some degree towards

154 weight-management. While weight-management apps are widely available in all stores and 155 in the largest app markets, use-patterns appear restricted. Consumers appear only to choose amongst a small number of the most downloaded apps: only 15 of almost 30,000 apps directed towards weight-management accounted for over half of all downloads. The US Institution of Medical Information recently reported similarly that nearly half of all mHealth apps downloads were for just 36 apps with the relatively low retention rates being at least $10 \%$ higher if the app has been advised by a healthcare professional ${ }^{13}$. Retention rate was also $30 \%$ higher for prescribed fitness apps and 30 -day retention rate was reports as $47 \%$ for health and fitness apps in $2012^{14}$.

Our results suggest that very few weight-management apps (0.05\%) were developed by official or professional sources, so quality might be of concern. Even those apps that were developed with professional input are not backed up by clinical studies on the effectiveness and validation of apps on health outcomes. Only three studies, to date, tested the effectiveness of commercially available apps on health outcomes with Direito's being the latest. He tested two commercially-available apps, aiming at increasing the physical activity of young people in New Zealand vs control over two months ${ }^{15}$. In this very small study, 51 participants were randomized but neither app significantly increased physical activity.

173 Few studies have assessed the quality of apps for controlling body-weight. Azar and 174 colleagues assessed 23 weight-loss apps for inclusion of behavioral theories using two 175 instruments: one on a traditional behavioural theory and the other on Fogg behavioural model. All assessed apps received low scores with both tools ${ }^{16}$. Pagoto and colleagues assessed 30 weight-loss apps and found that they included only $19 \%$ of 20 pre-defined behavioral strategies derived from an evidence-based weight-loss program ${ }^{17}$. Chen assessed weight-loss apps in Australia. Most of the 28 selected apps for quality assessment provided estimated energy requirements (86\%) and used a food database to calculate energy intake $(75 \%)^{18}$. Direito and colleagues assessed 40 apps from the New Zealand online stores: they included an average of 8.1 (range 2-18) behavioural-change techniques ${ }^{19}$. 
184 In view of the increased interest and activity in the mHealth sector, both the Food and Drug 185 Administration (FDA) in $\mathrm{US}^{20}$ and the European Commission (EC) ${ }^{21}$ have published guidelines

186 on mHealth apps. However, neither guideline provides any standards for the quality of app 187 content. FDA merely intends to exercise enforcement discretion for lifestyle apps, while EC 188 focuses on the legal framework for the sales of lifestyle and wellbeing apps.

190 An online study of this kind is inevitably limited by the quality of information presented by 191 the source material. Search terms may not identify all terminologies that may be used. It is 192 possible that more apps did involve some professional or official input, but if so that 193 information is not evident to potential users (public or professional). Healthcare industry and public organizations across the world have accelerated mHealth dialogues, to include more innovations including mobile/wireless technologies. However, to generate better health, and specifically better self-directed weight-control for obesity-prevention, there is need to incorporate more evidence-based methods into mHealth apps and reinforce movements like iPrescribeapps where apps are developed by medical experts for specific medical conditions ${ }^{22}$, especially since retention rates of health and fitness apps are so much better when those are prescribed instead of being self-selected. Researchers with evidence for effective online weight-loss or weight-gain prevention programmes in RCT settings could be encouraged to transfer their resources into an app form with greater reach. A new 'Apps For Patients' category, restricted to those with professional, evidence-based content, would be valuable, with subcategories for lifestyle-diseases, identifiable as for prevention or treatment.

In conclusion, mHealth offers potential to deliver improved, personalized, care while reducing healthcare-costs. For lifestyle-diseases and weight-management there are already many available apps but lack of professional, evidence-based content raises concerns about efficacy and patient/consumer safety. Encouraging app-development from tested and validated online-studies would offer confidence to both patients and healthcare-professionals. 
215 Supplementary information is available at IJO's website

216

217 
1. Yoo JH. The meaning of information technology (IT) mobile devices to me, the infectious disease physician. Infect Chemother. 2013;45(2):244-251.

2. Murfin M. Know your apps: an evidence-based approach to the evaluation of mobile clinical applications. J Physician Assist Educ. 2013;24(3):38-40.

3. Boulos MN, Wheeler S, Tavares $C$, Jones R. How smartphones are changing the face of mobile and participatory health care; an overview, with example from eCAALYX. Biomed Eng Online. 2011 Apr;10:24.

4. GSMA. 2015. The Mobile Economy. http://www.gsmamobileeconomy.com/GSMA Global Mobile Economy Report 20 15.pdf (accessed on 15/10/2015).

5. Wallace S, Clark M, White J. 'It's on my iPhone': attitudes to the use of mobile computing devices in medical education, a mixed-methods study. BMJ Open. 2012 Aug;2:e001099.

6. European Commision. Healthcare in your pocket: unlocking the potential of mHealth. 2014. http://europa.eu/rapid/press-release IP-14-394 en.htm (Accessed on 23/12/2015).

7. Payne KB, Wharrad $H$, Watts $K$. Smartphone and medical related app use among medical students and junior doctors in the United Kingdom (UK): a regional survey. BMC Med Inform Dec Mak. 2012 Oct;12:121.

8. NG M, Global, regional, and national prevalence of overweight and obesity in children and adults during 1980-2013: a systematic analysis for the Global Burden of Disease Study 2013 Lancet

9. Vlassopoulos A, Combet E, Lean ME. Changing distributions of body size and adiposity with age. Int J Obesity. 2014 Jun 1;38(6):857-64.

10. Health and Social Care Information Centre. Statistics on Obesity, Physical Activity and Diet- 2015. http://www.hscic.gov.uk/catalogue/PUB16988/obes-phys-acti-diet-eng2015.pdf (accessed on 25/05/2016).

11. Tang J, Abraham C, Greaves C, Yates T. Self-Directed Interventions to Promote Weight Loss: A Systematic Review of Reviews. Inter Medical Research. 2014. Feb;

12. $80 \%$ Of All Online Adults Now Own A Smartphone, Less Than $10 \%$ Use Wearables.2015. http://techcrunch.com/2015/01/12/80-of-all-online-adults-nowown-a-smartphone-less-than-10-use-wearables/ (accessed on

13. IMS Institute for Healthcare Informatics (2015). Patient Adoption of mHealth: Use, Evidence and Remaining Barriers to Mainstream Acceptance. IMS Health Incorporated.http://www.imshealth.com/files/web/IMSH\%20Institute/Reports/Pati ent\%20Adoption\%20of\%20mHealth/IIHI_Patient_Adoption_of_mHealth.pdf (accessed on 16/09/2016)

14. Farago P. App Engagement: The Matrix Reloaded. (2012) http://flurrymobile.tumblr.com/post/113379517625/app-engagement-the-matrixreloaded (accessed on 16/09/2016).

15. Direito A, Jiang Y, Whittaker R, Maddison R. Apps for IMproving FITness and Increasing Physical Activity Among Young People: The AIMFIT Pragmatic Randomized Controlled Trial. J Med Internet Res. 2015 Aug 27;17(8):e210. doi: 10.2196/jmir.4568. 
16. Azar KM, Lesser LI, Laing BY, Stephens J, Aurora MS, Burke LE, Palaniappan LP. Mobile applications for weight management: theory-based content analysis. Am J Prev Med. 2013 Nov;45(5):583-9. doi: 10.1016/j.amepre.2013.07.005.

17. Pagoto S, Schneider K, Jojic M, DeBiasse M, Mann D.Evidence-based strategies in weight-loss mobile apps. Am J Prev Med. 2013 Nov;45(5):576-82. doi: 10.1016/j.amepre.2013.04.025.

18. Chen J, Cade JE, Allman-Farinelli M. The Most Popular Smartphone Apps for Weight Loss: A Quality Assessment. JMIR Mhealth Uhealth. 2015 Dec 16;3(4):e104. doi: 10.2196/mhealth.4334.

19. Direito A, Dale LP, Shields E, Dobson R, Whittaker R, Maddison R.Do physical activity and dietary smartphone applications incorporate evidence-based behaviour change techniques? BMC Public Health. 2014 Jun 25;14:646. doi: 10.1186/1471-2458-14-646.

20. Food and Drug Administration organization (FDA). Mobile Medical Applications. Guidance for Industry and Food and Drug Administration Staff. 2015. http://www.fda.gov/downloads/MedicalDevices/.../UCM263366.pdf (Accessed on 21/12/2015).

21. European Commission. Commission staff working document on the existing legal EU framework applicable to lifestyle and wellbeing apps. 2014. file:///C:/ /Downloads/CommissionStaffWorkingDocumentontheexistingEUlegalframeworkapp licabletolifestyleandwellbeingapps.pdf. (accessed on 21/12/2015).

22. iPrescribeApps-From clinical problem to mobile solution. https://iprescribeapps.com/ (accessed on 25/05/2016). 
Figure 1: Main content of apps identified through the search in all five online app-stores 288

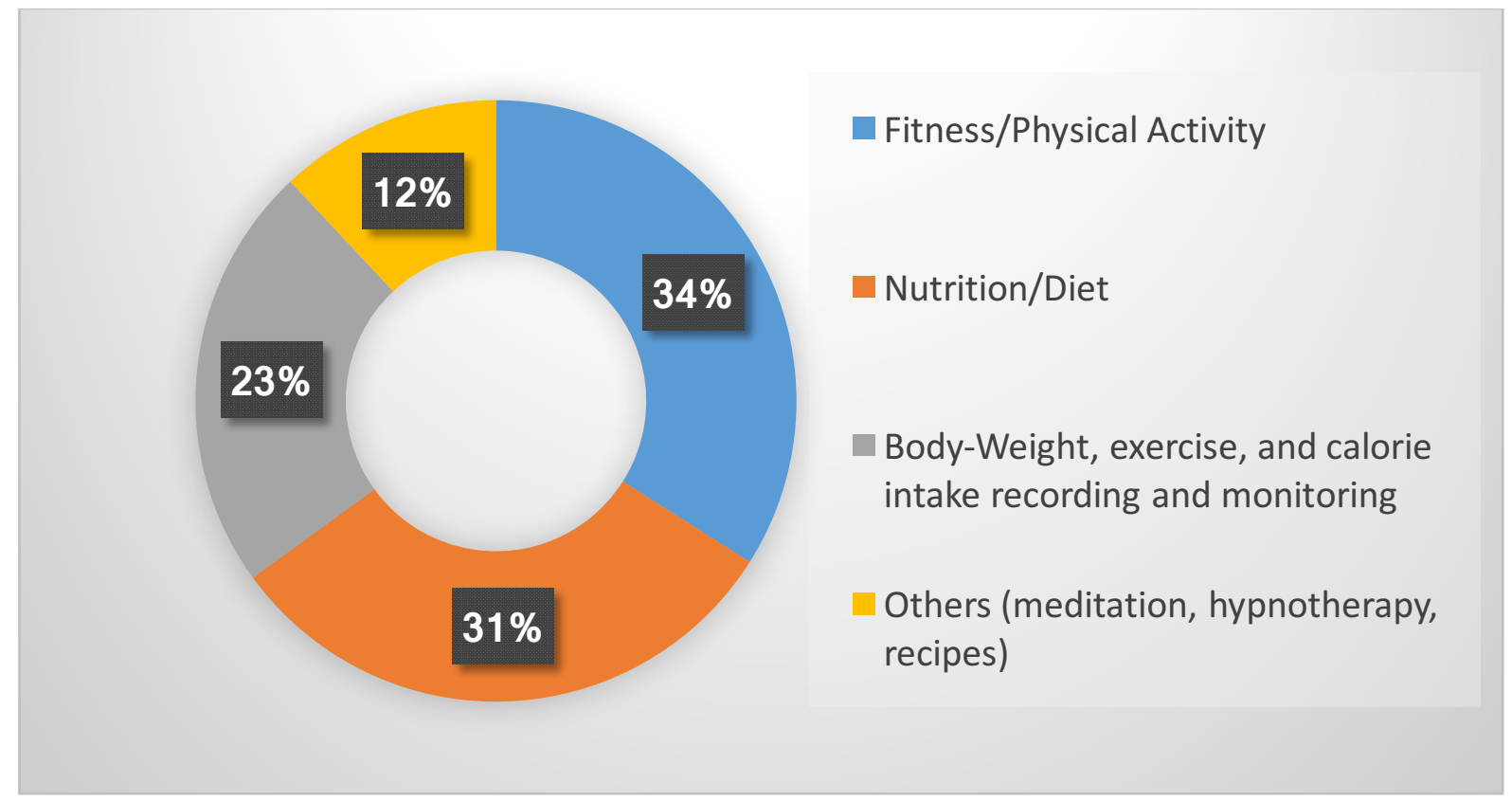

